\title{
Revealing the Environmental Dependence of Molecular Gas Content in a Distant X-Ray Cluster at $z=2.51$
}

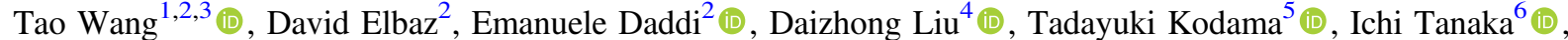

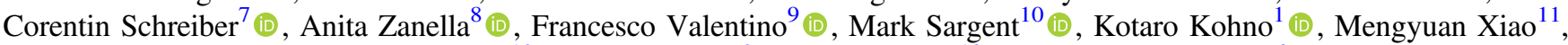 \\ Maurilio Pannella $^{12}$ (1) Laure Ciesla $^{2}$, Raphael Gobat ${ }^{13}$ (1) , and Yusei Koyama ${ }^{6}$ \\ ${ }^{1}$ Institute of Astronomy, Graduate School of Science, The University of Tokyo, 2-21-1 Osawa, Mitaka, \\ Tokyo 181-0015, Japan; taowang @ioa.s.u-tokyo.ac.jp, twang.nju@gmail.com \\ ${ }^{2}$ Laboratoire AIM-Paris-Saclay, CEA/DSM/Irfu, CNRS, Université Paris Diderot, Saclay, pt courrier 131, F-91191 Gif-sur-Yvette, France \\ ${ }^{3}$ National Astronomical Observatory of Japan, Mitaka, Tokyo 181-8588, Japan \\ ${ }^{4}$ Max Planck Institute for Astronomy, Königstuhl 17, D-69117 Heidelberg, Germany \\ ${ }^{5}$ Astronomical Institute, Tohoku University, Aramaki, Aoba-ku, Sendai 980-8578, Japan \\ ${ }^{6}$ Subaru Telescope, National Astronomical Observatory of Japan, National Institutes of Natural Sciences, 650 North A'ohoku Place, Hilo, HI 96720, USA \\ ${ }^{7}$ Leiden Observatory, Leiden University, NL-2300 RA Leiden, The Netherlands \\ ${ }^{8}$ European Southern Observatory, Karl Schwarzschild Str. 2, D-85748 Garching, Germany \\ ${ }^{9}$ Dark Cosmology Center, Niels Bohr Institute, University of Copenhagen, Juliane Maries Vej 30, DK-2100 Copenhagen, Denmark \\ ${ }^{10}$ Astronomy Centre, Department of Physics and Astronomy, University of Sussex, Brighton BN1 9QH, UK \\ ${ }^{11}$ Key Laboratory of Modern Astronomy and Astrophysics in Ministry of Education, School of Astronomy and Space Sciences, \\ Nanjing University, Nanjing, 210093, People's Republic of China \\ ${ }_{13}^{12}$ Faculty of Physics, Ludwig-Maximilians-Universität, Scheinerstr. 1, D-81679 München, Germany \\ ${ }^{13}$ Instituto de Física, Pontificia Universidad Católica de Valparaíso, Casilla 4059, Valparaíso, Chile \\ Received 2018 May 17; revised 2018 October 23; accepted 2018 October 23; published 2018 November 8
}

\begin{abstract}
We present a census of the molecular gas properties of galaxies in the most distant known X-ray cluster, CLJ1001, at $z=2.51$, using deep observations of $\mathrm{CO}(1-0)$ with the Karl G. Jansky Very Large Array. In total, 14 cluster members with $M_{*}>10^{10.5} M_{\odot}$ are detected, including all of the massive star-forming members within the virial radius, providing the largest galaxy sample in a single cluster at $z>2$ with $\mathrm{CO}(1-0)$ measurements. We find a large variety in the gas content of these cluster galaxies, which is correlated with their relative positions (or accretion states), with those closer to the cluster core being increasingly gas-poor. Moreover, despite their low gas content, the galaxies in the cluster center exhibit an elevated star formation efficiency (SFE; SFE $=\mathrm{SFR} / M_{\mathrm{gas}}$ ) compared to field galaxies, suggesting that the suppression on the SFR is likely delayed compared to that on the gas content. Their gas depletion time is around $t_{\mathrm{dep}} \sim 400 \mathrm{Myr}$, which is comparable to the cluster dynamical time. This implies that they will consume all of their gas within a single orbit around the cluster center, and form a passive cluster core by $z \sim 2$. This result is one of the first direct pieces of evidence for the influence of environment on the gas reservoirs and SFE of $z>2$ cluster galaxies, thereby providing new insights into the rapid formation and quenching of the most massive galaxies in the early universe.
\end{abstract}

Key words: galaxies: clusters: general - galaxies: formation - galaxies: high-redshift - galaxies: ISM

\section{Introduction}

Galaxy clusters in the present-day universe are dominated by a population of massive quiescent galaxies in their center (Dressler et al. 1997). The formation mechanisms of these massive galaxies and the influence that environment plays in this process remain open questions. These issues are difficult to address in the local universe, as most of the massive galaxies have already been in place for $10 \mathrm{Gyr}$ and signatures of their formation history have been largely erased. Contrary to mature clusters at low redshifts, a significant population of (proto) clusters with active star formation has been found at $z>2-4$, the peak formation epoch of massive cluster galaxies (Thomas et al. 2005). With a large number of massive star-forming galaxies (SFGs) in a cluster-like environment, these structures provide ideal laboratories to explore the environmental dependence of massive galaxy formation.

The different properties, for example, the star formation rate (SFR) of galaxies in $z>2$ (proto-)clusters and field, have been extensively studied. However, no significant difference has been found on the average SFRs, i.e., the normalization of the star-forming main sequence (MS), at $z \sim 2$ (Koyama et al. 2013;
Shimakawa et al. 2017). A few studies show an enhanced fraction of starburst galaxies in (proto-)clusters at both the bright (Casey 2016; Wang et al. 2016) and faint end of the stellar mass function (Hayashi et al. 2016), however, statistical samples are still required to confirm these findings. Overall, this lack of strong environmental dependence of star formation may indicate that there is a significant delay between the first infall of cluster galaxies and substantial reduction in their SFRs, which likely only take place close to the cluster core (Wetzel et al. 2013).

While it is not yet fully clear how SFR depends on environment, ample evidence exists for the deficit of cold gas, the fuel of star formation, for galaxies in dense environments (Boselli \& Gavazzi 2006). Both neutral hydrogen (HI), molecular gas, and even dust in cluster galaxies can be severely impacted by their local environment through, for example, ram pressure stripping and tidal stripping, at least in the vicinity of the cluster core (Cortese et al. 2010; Davis et al. 2013; Jáchym et al. 2014, 2017). On the other hand, while pioneering studies on molecular gas content in high- $z$ clusters $(z \sim 1.5-2)$ have been recently performed, there is still no consensus on the influence of environment on galaxies' gas content (Aravena et al. 2012; 
Wagg et al. 2012; Casasola et al. 2013; Dannerbauer et al. 2017; Noble et al. 2017; Stach et al. 2017; Coogan et al. 2018). This is mainly driven by the limited number of detections, which is often biased toward the most gas-rich members except for a few cases (Hayashi et al. 2017; Rudnick et al. 2017). In addition, most of these targeted clusters appear to be already dominated by massive quiescent galaxies in the core, but little is known about their gas and star formation properties during the epoch of their formation/quenching. Though studies of molecular gas properties in dense environments exist at $z>2$, they have only detected some of the brightest member galaxies (e.g., Tadaki et al. 2014; Dannerbauer et al. 2017; Lee et al. 2017, and references therein), inhibiting a comprehensive understanding of the gas content of cluster galaxies.

In this Letter, we present a census of molecular gas properties of 14 massive star-forming galaxies (SFGs) in the most distant known X-ray cluster, CLJ1001, at $z=2.51$ (Wang et al. 2016, hereafter, W16), based on $\operatorname{CO}(1-0)$ observations with the Karl G. Jansky Very Large Array (JVLA). CLJ1001 is estimated to have total mass of $M \sim 10^{13.9 \pm 0.2} M_{\odot}$ and virial radius of $R_{200 c} \sim 340 \mathrm{kpc}$ based on its X-ray emission and velocity dispersion (W16). Our recent deep narrowband (NB) imaging further reveals a large number of $\mathrm{H} \alpha$-emitters at $z=2.51$ in the cluster, providing further evidence that this is a different structure with respect to the protocluster/large-scale structure found, in the same region of the sky, by Casey et al. (2015) at $z=2.47$. Despite its extended X-ray emission, this cluster is dominated by massive SFGs in the core, which are all detected in $\mathrm{CO}(1-0)$, allowing us, for the first time, to probe the gas content and star formation efficiency (SFE) for a complete sample of massive cluster members (down to $M_{*}>10^{10.5} M_{\odot}$ ) at $z>2$. Throughout the Letter, we assume cosmological parameters of $H_{0}=70 \mathrm{~km} \mathrm{~s}^{-1} \mathrm{Mpc}^{-1}, \Omega_{M}=0.3$, and $\Omega_{\Lambda}=0.7$. A Chabrier (2003) initial mass function is adopted to derive stellar masses and SFRs.

\section{Observations}

\subsection{JVLA CO(1-0) Observations}

Our JVLA observations of CLJ1001 were performed in 2015 December under the program 15B-290 (P.I.: Tao Wang). Part of the data has been already presented in W16 (including example $\mathrm{CO}(1-0)$ spectra), which were only used to confirm cluster members. In detail, the observations were carried out in the $K a$-band with the $\mathrm{D}$ configuration, with an effective frequency coverage of $32.2-33.59 \mathrm{GHz}$, corresponding to $z \sim 2.43-2.58$ for $\mathrm{CO}(1-0)$. The observations were done in excellent weather conditions with precipitable water vapor as low as $2.0 \mathrm{~mm}$ and wind speed $0.3-1.5 \mathrm{~km} \mathrm{~s}^{-1}$, therefore the achieved system temperature $\left(T_{\text {sys }}\right)$ is about or even below $40 \mathrm{~K}$, while typical $T_{\text {sys }}$ is about $50 \mathrm{~K}$ at the $K a$-band in winter. The full width half power (FWHP) size of the primary beam is 1.3 at $32.878 \mathrm{GHz}(z=2.506$ for $\mathrm{CO}(1-0))$. We observed $3 \mathrm{C} 147$ for flux calibration during the full observations, and a point source J1024-0052 near our target for phase calibration during each scan loop (every $\sim 8$ minutes). The total integration time is $\sim 13 \mathrm{hr}$. The data were reduced using the Common Astronomy Software Application (CASA) package (McMullin et al. 2007) with a standard pipeline. We chose 0.15 pixels and a spectral resolution of $30 \mathrm{~km} \mathrm{~s}^{-1}$ with a natural weighting scheme for imaging. Image deconvolution was performed with a CLEAN threshold of $3 \sigma$ of each cube. The resulting datacube has a synthesized beam size of $\sim 2$ !" $88 \times 2$ " 52 with an $\mathrm{rms}$ of $\sim 33-40 \mu \mathrm{Jy}_{\text {beam }}{ }^{-1}$ per channel at the phase center.

\subsection{Subaru/MOIRCS NB Imaging}

In order to acquire a complete census of cluster (starforming) members, we recently conducted a deep NB survey toward CLJ1001 with Subaru/MOIRCS. The NB survey employed the "CO" filter centered at $2.3 \mu \mathrm{m}$ to identify $\mathrm{H} \alpha$ emitters at $z=2.49-2.52$, combined with the already available deep $K_{s}$-band data in COSMOS from the UltraVista survey (McCracken et al. 2012; Muzzin et al. 2013; Laigle et al. 2016). With $4.4 \mathrm{hr}$ of integration, we have detected $49 \mathrm{H} \alpha$ emitters with line flux down to $1.5 \times 10^{-17} \mathrm{erg} \mathrm{s}^{-1} \mathrm{~cm}^{-2}$. This corresponds to a dust-free SFR of $\sim 5 M_{\odot} \mathrm{yr}^{-1}$ at $z=2.51$ (Kennicutt 1998). Details of data reduction and star formation properties of these $\mathrm{H} \alpha$ emitters will be discussed in a forthcoming paper. Here we only use their positions to search for $\mathrm{CO}(1-0)$ line emissions.

\subsection{Extraction of $\mathrm{CO}(1-0)$ Emitters}

We extract $\mathrm{CO}(1-0)$ spectra at the position of the cluster members (H $\alpha$-emitters) out to $2 \times$ FWHP of the primary beam (PB). This approach allows us to detect sources with fainter fluxes and with higher fidelity than a blind search. In total, $14 \mathrm{H} \alpha$-emitters are detected with a signal-to-noise ratio $(\mathrm{S} / \mathrm{N})>3$ (Figure 1). We measured the $\mathrm{CO}(1-0)$ line fluxes for each object by running a 2D Gaussian fit with CASA (IMFIT) on the velocity-integrated (moment-0) map. The velocity range used to create the moment- 0 map of each object was determined so to maximize the signal-to-noise of the detection. During this process the spatial position of the targets was kept fixed based on the coordinates found from the Hubble Space Telescope (HST)/F160W (if available) or NB ancillary images, in order to minimize false detections due to noise fluctuations. Thirteen out of these 14 galaxies (except ID-14) are also covered by our Atacama Large Millimeter/submillimeter Array (ALMA) band-3 observations, and they are all detected in $\mathrm{CO}(3-2)$ at roughly the same velocity (Wang et al. 2018, in preparation). In the case of low $\mathrm{S} / \mathrm{N}$ with $\mathrm{CO}(1-0), \mathrm{CO}(3-2)$ data is combined with $\mathrm{CO}(1-0)$ to determine the velocity range of the line emission. For a sanity check, we have also measured directly their total fluxes in the $u v$-plane with GILDAS, ${ }^{14} \mathrm{a}$ procedure that gives consistent results. The measured integrated $\mathrm{CO}(1-0)$ line intensities, after primary beam correction, are listed in Table 1 . The 14 detections include all but 1 massive galaxy (ID-131651 in W16) with $M_{*}>10^{10.5} M_{\odot}$ within the $\mathrm{PB}$, which is classified as a passive galaxy in W16 based on its rest-frame colors, suggesting that the $\mathrm{H} \alpha$ emission mostly likely originates from an (radio) active galactic nucleus (AGN), as further supported by the non-detection of $\mathrm{CO}(1-0)$.

\subsection{Molecular Gas Masses from CO(1-0)}

The use of $\mathrm{CO}(1-0)$ avoids the uncertainty in the $\mathrm{CO}$ excitation, and is the most extensively used way for obtaining the total molecular gas mass, $M\left(\mathrm{H}_{2}\right)$. The conversion involves the integrated $\mathrm{CO}$ emission intensity $\left(L_{\mathrm{CO}(1-0)}^{\prime}\right)$ and a conversion factor $\alpha_{\mathrm{CO}}$ (Bolatto et al. 2013), through $M\left(\mathrm{H}_{2}\right)=\alpha_{\mathrm{CO}} L_{\mathrm{CO}}^{\prime}$, with the $\mathrm{CO}$ line luminosity $L_{\mathrm{CO}(1-0)}^{\prime}$ derived following Solomon \& Vanden Bout (2005). We determine $\alpha_{\mathrm{CO}}$ for the cluster galaxy

\footnotetext{
${ }^{14}$ http://www.iram.fr/IRAMFR/GILDAS
} 

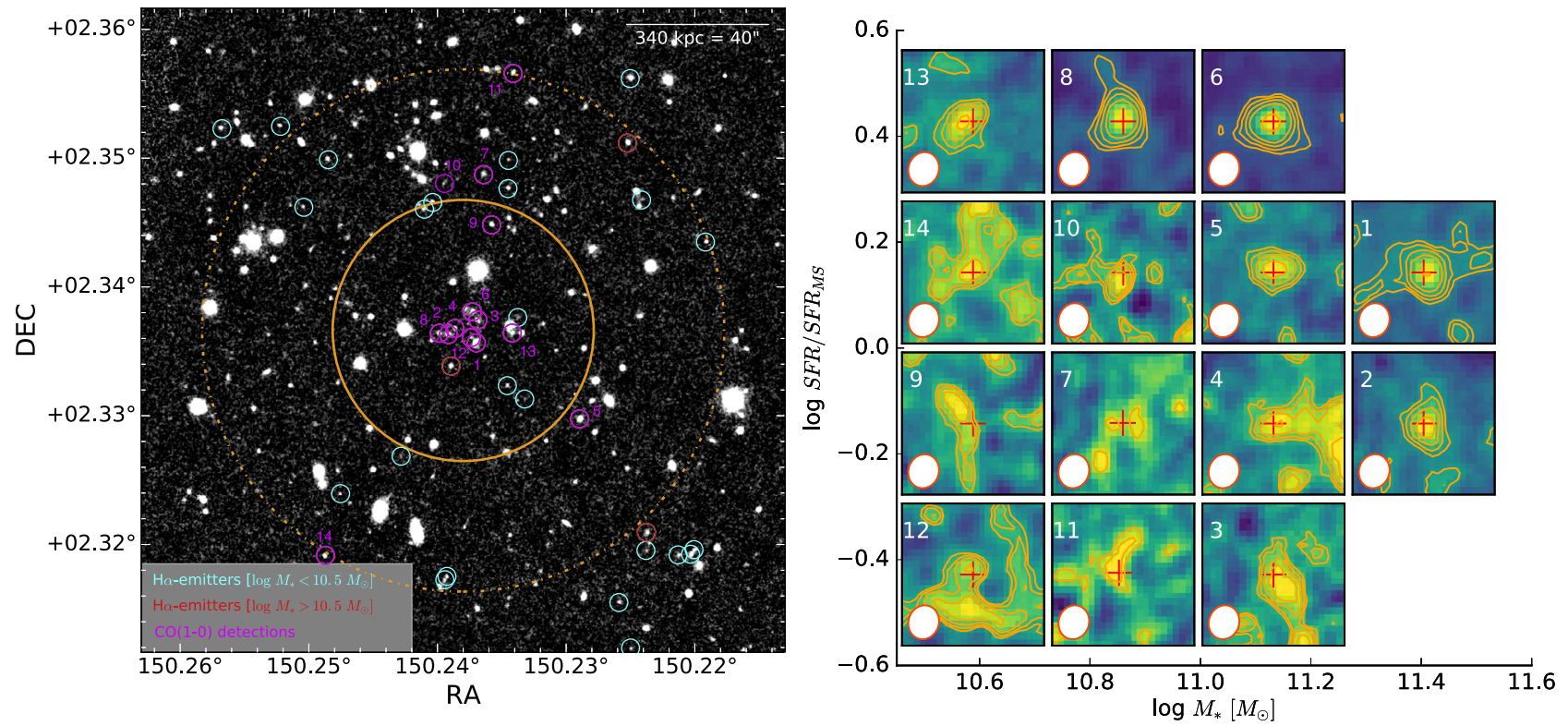

Figure 1. Left panel: sky distributions of cluster members around the core of CLJ1001. The background image is the NB image from MOIRCS/Subaru at $2.3 \mu \mathrm{m}$. The cyan and red open circles denote low- and high-mass star-forming cluster members (H $\alpha$ emitters) at $z=2.51$ separated at $M_{*}=10^{10.5} M_{\odot}$. The $\mathrm{CO}(1-0)$ detected members all have $M_{*}>10^{10.5} M_{\odot}$, and are further indicated in magenta. The scale bar indicates the virial radius $\left(R_{200 c}\right)$ of the cluster. The small and large orange circles denote the coverage of the JVLA observations, with the diameter corresponding to 1 and $2 \times$ FWHP of the primary beam at $32.878 \mathrm{GHz}$, respectively. Right panel: the velocity-integrated intensity map (moment- 0 ) of CO $J=1-0$ for the 14 galaxies detected by JVLA. The position of each panel is determined by their stellar mass and SFR (normalized by the SFR of MS galaxies at the same mass). Each panel is $12^{\prime \prime} \times 12^{\prime \prime}$. Contour levels of CO(1-0) starts at $\sqrt{2} \sigma$ and increase as 2, 2 $\sqrt{2}, 4$, 8 , and $16 \sigma$. The red cross in each panel indicates the centroid of the stellar emission as determined from the HST/F160W (if available) or NB images. The derived integrated fluxes are presented in Table 1 .

Table 1

Physical Properties of the CO(1-0)-detected Cluster Members in CLJ1001

\begin{tabular}{|c|c|c|c|c|c|c|c|c|c|}
\hline ID & $\mathrm{ID}^{\mathrm{a}}(\mathrm{W} 16)$ & $z_{\mathrm{CO}}$ & $\begin{array}{c}\log M_{*} \\
\left(M_{\odot}\right)\end{array}$ & $\begin{array}{c}\log L_{\mathrm{IR}} \\
\left(L_{\odot}\right)\end{array}$ & $\begin{array}{c}\text { FWHM } \\
\left(10^{2} \mathrm{~km} \mathrm{~s}^{-1}\right)\end{array}$ & $\begin{array}{c}L_{\mathrm{CO}(1-0)}^{\prime} \\
\left(10^{10} \mathrm{~K} \mathrm{~km} \mathrm{~s}^{-1} \mathrm{pc}^{2}\right)\end{array}$ & $\begin{array}{c}\alpha_{\mathrm{CO}} \\
\left(M_{\odot} /\left(\mathrm{K} \mathrm{km} \mathrm{s}^{-1} \mathrm{pc}^{2}\right)\right)\end{array}$ & $\begin{array}{c}M_{\text {gas }} \\
\left(10^{10} M_{\odot}\right)\end{array}$ & $\begin{array}{c}t_{\mathrm{dep}} \\
(\mathrm{Gyr})\end{array}$ \\
\hline 1 & 130949 & 2.503 & $11.36 \pm 0.15$ & $12.55 \pm 0.14$ & $5.0 \pm 0.5$ & $2.3 \pm 0.2$ & 4.06 & $9.2 \pm 0.9$ & 0.26 \\
\hline 2 & 130901 & 2.507 & $11.35 \pm 0.15$ & $12.04 \pm 0.21$ & $6.8 \pm 0.9$ & $1.8 \pm 0.3$ & 4.06 & $7.4 \pm 1.1$ & 0.68 \\
\hline 3 & 131079 & 2.514 & $11.13 \pm 0.15$ & $11.88 \pm 0.20$ & $2.8 \pm 1.2$ & $0.6 \pm 0.1$ & 4.08 & $2.4 \pm 0.3$ & 0.32 \\
\hline 4 & 130933 & 2.501 & $11.06 \pm 0.15$ & $12.09 \pm 0.16$ & $6.9 \pm 1.6$ & $0.6 \pm 0.1$ & 4.08 & $2.6 \pm 0.5$ & 0.21 \\
\hline 5 & 130359 & 2.508 & $11.03 \pm 0.15$ & $12.44 \pm 0.13$ & $2.4 \pm 0.4$ & $2.7 \pm 0.4$ & 4.08 & $11.1 \pm 1.5$ & 0.40 \\
\hline 6 & 131077 & 2.494 & $10.93 \pm 0.15$ & $12.87 \pm 0.13$ & $5.5 \pm 0.4$ & $4.9 \pm 0.4$ & 4.09 & $20.2 \pm 1.7$ & 0.27 \\
\hline 7 & 132044 & 2.505 & $10.90 \pm 0.15$ & $12.08 \pm 0.18$ & $6.8 \pm 1.6$ & $2.8 \pm 0.8$ & 4.09 & $11.3 \pm 3.5$ & 0.94 \\
\hline 8 & 130891 & 2.513 & $10.83 \pm 0.15$ & $12.62 \pm 0.13$ & $3.4 \pm 0.2$ & $3.2 \pm 0.3$ & 4.10 & $13.3 \pm 1.4$ & 0.32 \\
\hline 9 & 131661 & 2.500 & $10.80 \pm 0.15$ & $11.97 \pm 0.19$ & $0.9 \pm 0.4$ & $0.5 \pm 0.1$ & 4.10 & $1.9 \pm 0.3$ & 0.20 \\
\hline 10 & 131904 & 2.506 & $10.80 \pm 0.15$ & $11.78 \pm 0.25$ & $6.9 \pm 3.7$ & $1.0 \pm 0.3$ & 4.10 & $4.2 \pm 1.1$ & 0.70 \\
\hline 11 & 132627 & 2.506 & $10.73 \pm 0.15$ & $12.27 \pm 0.16$ & $6.0 \pm 1.9$ & $12.7 \pm 4.2$ & 4.10 & $52.2 \pm 17.4$ & 2.83 \\
\hline 12 & 130842 & 2.515 & $10.67 \pm 0.15$ & $11.10 \pm 0.20$ & $0.9 \pm 0.3$ & $0.3 \pm 0.1$ & 4.11 & $1.4 \pm 0.3$ & 1.07 \\
\hline 13 & $\cdots$ & 2.505 & $10.67 \pm 0.15$ & $12.31 \pm 0.20$ & $5.3 \pm 0.8$ & $2.0 \pm 0.3$ & 4.11 & $8.4 \pm 1.3$ & 0.41 \\
\hline 14 & 129444 & 2.515 & $10.54 \pm 0.15$ & $11.95 \pm 0.25$ & $1.4 \pm 0.3$ & $13.4 \pm 2.7$ & 4.12 & $55.0 \pm 11$ & 6.17 \\
\hline
\end{tabular}

Note.

${ }^{\text {a }}$ IDs are from the $K_{s}$-selected catalog in Muzzin et al. (2013).

sample following Genzel et al. (2015) and Tacconi et al. (2018). The same mass-metallicity relation used in Genzel et al. (2015) is also applied to determine the metallicity for the CO-detected galaxies in our sample, which is close to solar given their large stellar masses. As a result, the derived $\alpha_{\mathrm{CO}}$ is close to the Milky value for this sample (Table 1).

\section{Results}

\section{1. $S F E$}

The combination of the $\mathrm{CO}$ line luminosity $\left(L_{\mathrm{CO}(1-0)}^{\prime}\right)$, tracing total molecular gas, and the total infrared (IR) luminosity
$\left(L_{\mathrm{IR}}\right)$, tracing newly formed stars, provides a crucial constraint on the SFE, with $L_{\mathrm{IR}} / L_{\mathrm{CO}(1-0)}^{\prime} \propto \mathrm{SFR} / M_{\mathrm{gas}} \equiv \mathrm{SFE}$. Moreover, the use of $L_{\mathrm{IR}} / L_{\mathrm{CO}(1-0)}^{\prime}$ as an approximation of SFE allows us to not have to account for the different prescriptions for the CO-to- $\mathrm{H}_{2}$ conversion factor, enabling direct comparisons between different samples. As shown in W16 (also see, e.g., Bussmann et al. 2015), five cluster members are detected at $870 \mu \mathrm{m}$ with ALMA, for which we derive their IR luminosities, $L_{\mathrm{IR}}$, by fitting the full-IR SED. For the other galaxies without $870 \mu \mathrm{m}$ detection, we derive $L_{\mathrm{IR}}$ based on their $24 \mu \mathrm{m}$ fluxes (Muzzin et al. 2013) and $3 \mathrm{GHz}$ (Smolčić et al. 2017) radio continuum by using the average IR SED templates for 

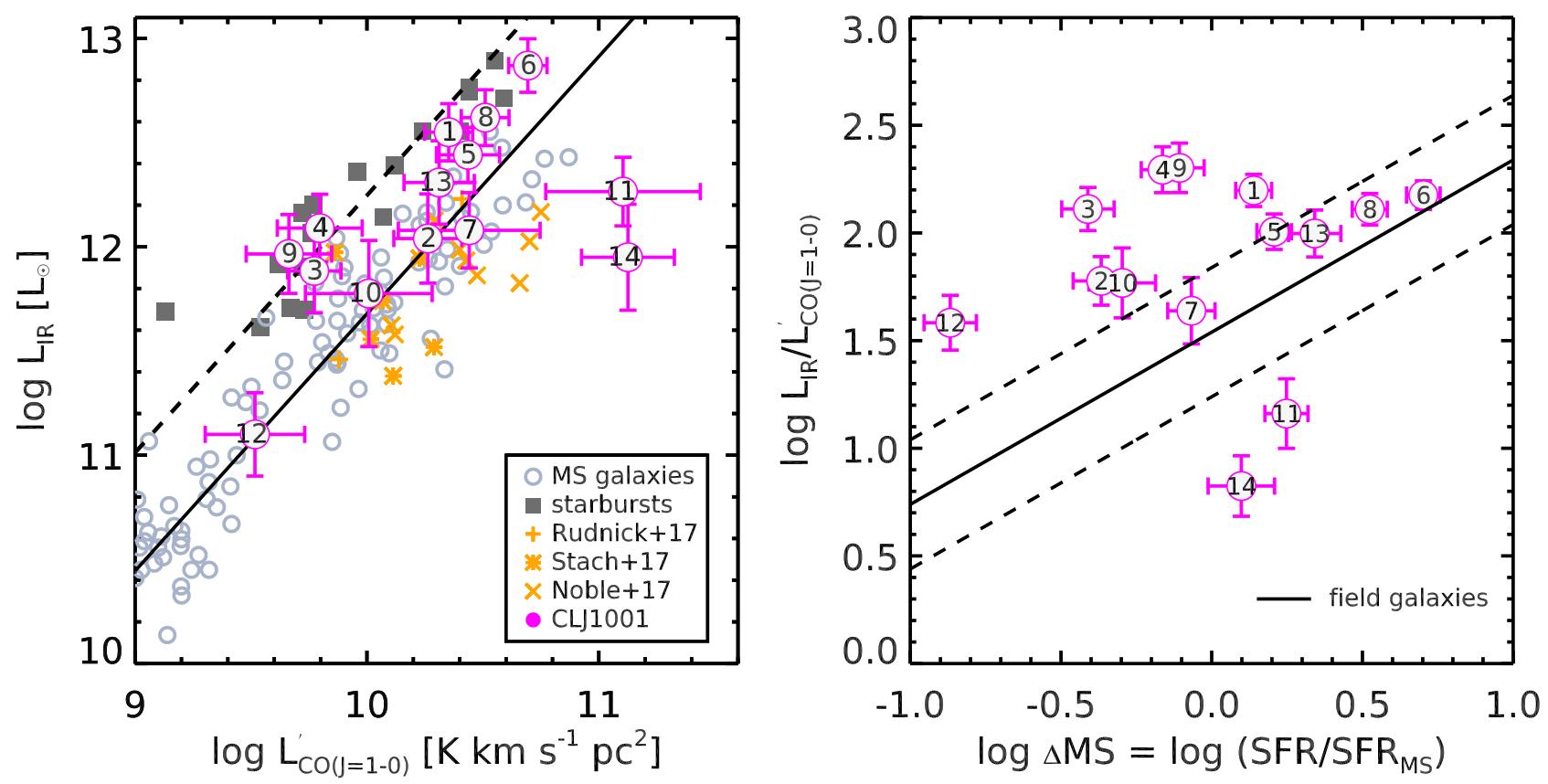

Figure 2. Left panel: IR luminosities, $L_{\mathrm{IR}}$, vs. $\mathrm{CO}(1-0)$ line luminosities, $L_{\mathrm{CO}(1-0)}^{\prime}$, for $\mathrm{CO}(1-0)$ detected galaxies in CLJ1001. The solid and dashed lines represent the scaling relation for field MS and strong SB galaxies ( $\triangle \mathrm{MS} \sim 10$ ), respectively (Sargent et al. 2014). The field galaxy sample includes both MS and SB galaxies drawn from the compilation by Sargent et al. (2014), and a sample of $z \sim 1$ SB galaxies by Silverman et al. (2015). We also include a few recent studies of cluster galaxies at $z \gtrsim 1.5$, which have either CO(2-1) (Stach et al. 2017; Noble et al. 2017) or CO(1-0) (Rudnick et al. 2017) measurements. Right panel: SFE, as indicated by the ratio between $L_{\mathrm{IR}}$ and $L_{\mathrm{CO}(1-0)}^{\prime}$, vs. $\Delta \mathrm{MS}$ for galaxies in CLJ1001. The best-fit relation for field galaxies and its associated $1 \sigma$ scatter are also shown (Magdis et al. 2012). A large variety of SFE for cluster galaxies is observed.

galaxies at $z \sim 2.5$ (Schreiber et al. 2018) and far-infrared (FIR)radio relation (Delhaize et al. 2017). We have verified this approach through comparisons of IR-SED derived and $3 \mathrm{GHz}$ derived $L_{\mathrm{IR}}$ for four out of the five ALMA-detected sources (excluding ID-4, which is a radio AGN), which are in good agreement. Only one source (ID-12) has neither $24 \mu \mathrm{m}$ nor $3 \mathrm{GHz}$ detections, for which we derive $L_{\mathrm{IR}}$ based on its SFR estimated from extinction-corrected $\mathrm{H} \alpha$ following Kennicutt (1998). The best-estimated $L_{\mathrm{IR}}$ for cluster members are listed in Table. 1.

Figure 2 presents the comparison of $L_{\mathrm{IR}}$ and $L_{\mathrm{CO}(1-0)}^{\prime}$ between member galaxies in CLJ1001 and other galaxy populations in high- $z$ clusters $(z \sim 1.5-2)$ and field. The CLJ1001 galaxies exhibit a large variety in their SFE as traced by $L_{\mathrm{IR}} / L_{\mathrm{CO}(1-0)}^{\prime}$, including members with high, starburst-like SFE and also members with SFE even below MS-like galaxies. To examine whether this large variety in SFE is solely driven by their different star formation modes (starburst versus MS), we further show the variation of $L_{\mathrm{IR}} / L_{\mathrm{CO}(1-0)}^{\prime}$ as a function of their distance to the MS, which is defined as $\Delta \mathrm{MS}=\mathrm{SFR} / \mathrm{SFR}_{\mathrm{MS}}$ with $\mathrm{SFR}_{\mathrm{MS}}$ for each galaxy derived using the MS relation at $z=2.5$ (Schreiber et al. 2015) at the same stellar mass (the right panel of Figure 2), which is nearly identical to the MS relation used in Tacconi et al. (2018). Consistent with field galaxies, a general trend toward increasing SFE with enhanced star formation activity (relative to the MS) is observed in CLJ1001. However, in contrast to field galaxies, a large dispersion of SFE is present for these cluster galaxies. Most prominently, a population of cluster galaxies with MS-like SFR $(\Delta M \mathrm{MS} \mid<0.5)$ exhibit significantly different SFE compared to field galaxies. This suggests that the large variation in SFE for these cluster galaxies is not driven by the variation in SFR; instead, some other mechanisms, most likely related to the dense environment, may play an important role.

\subsection{Clustercentric Radius Dependence of Star Formation and Molecular Gas Content}

In order to gain further insights into the origin of the gas and star formation properties of the cluster galaxies, we examine the relation between these properties and their positions in the cluster. Specifically, we employ the line-of-sight velocity versus clustercentric radius phase-space diagram to illustrate the relative distribution of member galaxies within the cluster. The phase-space diagram characterizes the accretion state of cluster member galaxies, which minimizes projection effects of their 2D positions with respect to the cluster center (see, e.g., Noble et al. 2013). In this diagram, galaxies that are recently accreted to the cluster tend to have large relative velocities and/or large clustercentric radius, which are offset from the central virialized region. As shown in the left panel of Figure 3, we observe a clear trend of decreasing gas content $\left(\mu_{\text {gas }}=M_{\text {gas }} / M_{*}\right)$ with proximity to the cluster core. This is more clearly illustrated in the right panel of Figure 3, in which we plot $\mu_{\text {gas }}$ versus $k_{b}=\left(|\Delta \nu| / \sigma_{\text {cluster }}\right) \times\left(R / R_{200 \mathrm{c}}\right)$ for the cluster galaxies. The parameter $k_{b}$ converts the phase-space diagram into one dimension (Noble et al. 2013). Galaxies with lower $k_{b}$ are more closely bounded to the cluster, hence are likely accreted at earlier times. Figure 3 reveals clearly a trend that galaxies with high gas fraction (relative to the MS) have entered the cluster more recently than the gas-poor members. This remains true even when the normalized gas fraction, $\mu_{\mathrm{gas}} / \mu_{\mathrm{gas}, \Delta \mathrm{MS}}$, is adopted. While galaxies in the outskirts of the cluster exhibit a large scatter in their gas fraction (compared to field galaxies), galaxies in the cluster center $\left(k_{b} \lesssim 0.1\right)$ 


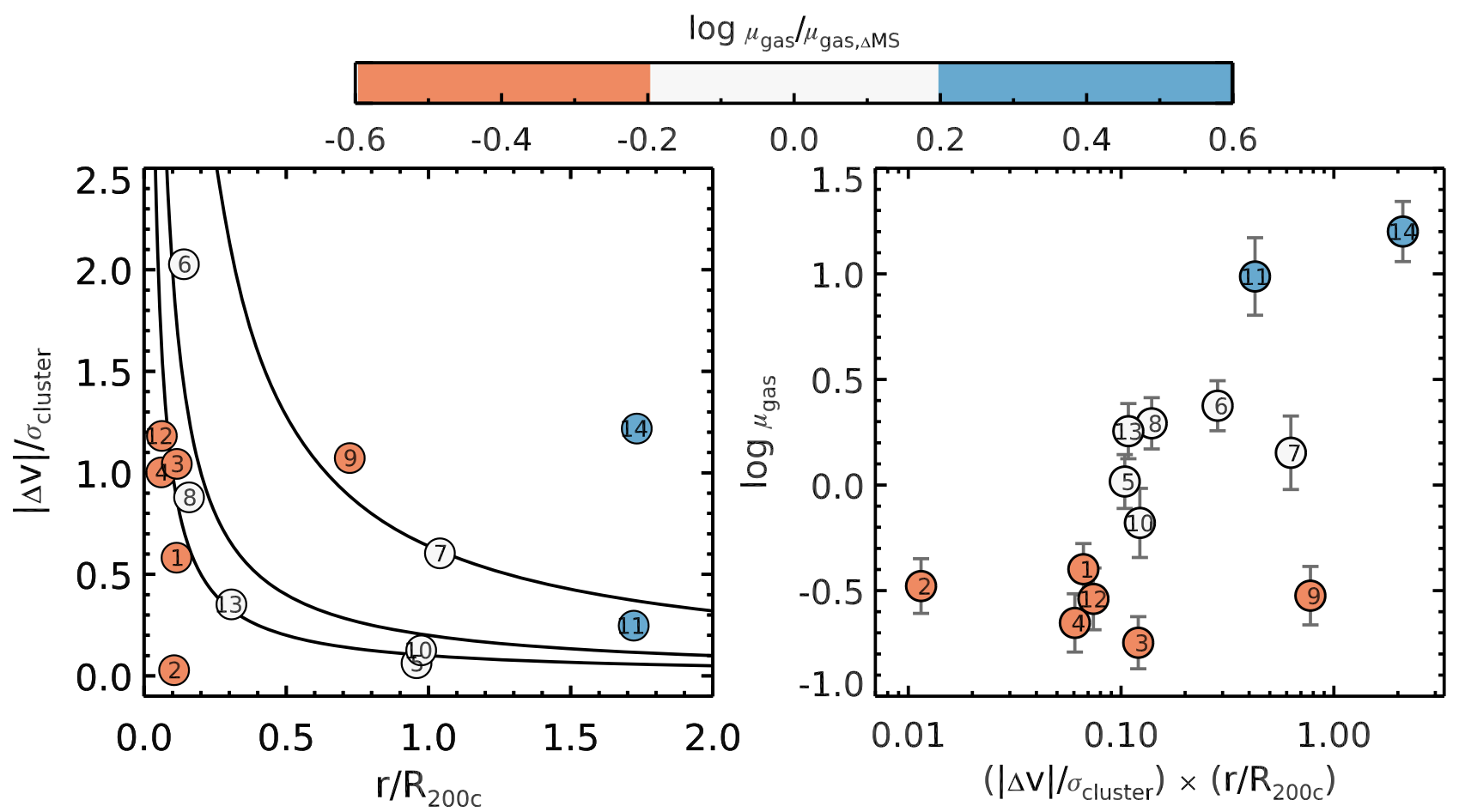

Figure 3. Clustercentric-radius dependence of gas fraction as shown in the phase-space diagram (left panel) and gas fraction vs. $k_{b}=\left(|\Delta v| / \sigma_{\text {cluster }}\right) \times\left(R / R_{200 c}\right)$ plane, which is a proxy for clustercentric radius in 3D (right panel). Galaxies are color-coded by their gas fraction normalized by the value of field galaxies at the same $\Delta \mathrm{MS}$, mass, and redshifts (Tacconi et al. 2018). Curves of constant $k_{b}$ values with $k_{b}=0.05,0.2,0.64$ are shown in the left panel. A strong clustercentric radius dependence of gas content is revealed, with decreasing gas fraction for galaxies closer to the cluster center, which is true for either absolute or normalized value of gas fraction.

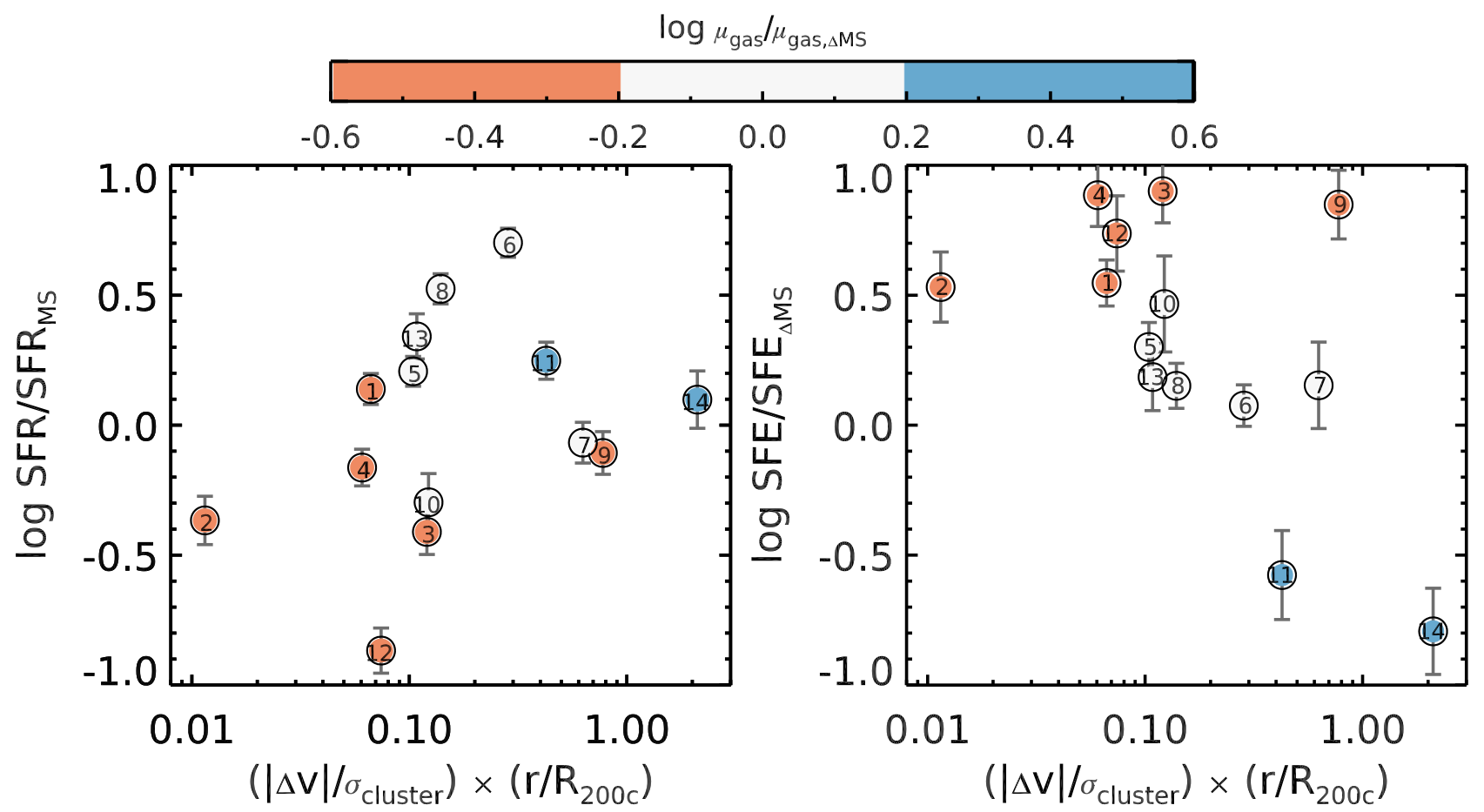

Figure 4. Dependence of SFR (left panel) and SFE (right panel) on the clustercentric radius indicated by $k_{b}=\left(|\Delta v| / \sigma_{\text {cluster }}\right) \times\left(R / R_{200 \mathrm{c}}\right)$. The SFR of each galaxy is normalized by field MS galaxies, while the SFE is normalized by field galaxies at the same $\Delta$ MS (Magdis et al. 2012; Tacconi et al. 2018). Galaxies are color-coded by their gas fraction normalized by the field galaxies at the same $\triangle \mathrm{MS}$. A general trend of enhanced SFE toward the cluster center is revealed. The low gas fraction, yet normal or suppressed SFR of the member galaxies in the cluster center, suggests that their enhanced SFE is mainly caused by their deficit of molecular gas (instead of an enhanced SFR).

show exclusively a deficit of molecular gas. The transition between the gas-rich and gas-poor populations takes place at around $k_{b} \sim 0.1$. This rapid transition may suggest that whatever environmental effects are involved, this process must be very efficient in reducing the gas content of cluster galaxies. 
We further present the variation of SFR and SFE as a function of $k_{b}$ in Figure 4, showing that both SFR and SFE also varies with clustercentric radius. While galaxies in the outskirts of the cluster exhibit a large scatter in their SFR, most member galaxies in the center tend to fall below the MS. On the other hand, these galaxies in the center show a significant enhancement in their SFE compared to those in the outskirts and field galaxies. Despite their low gas fraction, their normal or suppressed SFR suggests that their enhanced SFE is mainly caused by their deficit of molecular gas (instead of an enhanced SFR). This indicates that the suppression on the SFR from the dense environment is likely delayed compared to that on the gas content. This high SFE ensures that most of these galaxies will likely consume all of their gas in a short timescale. Their gas depletion time $\left(t_{\mathrm{dep}}=1 / \mathrm{SFE}\right)$ is around $t_{\mathrm{dep}} \sim 0.4 \mathrm{Gyr}$, a factor of two shorter than field galaxies with the same $\Delta \mathrm{MS}$ (Figure 2). This timescale is comparable to the cluster dynamical time (approximated by the crossing time), $t_{\text {dyn }} \sim R_{200 \mathrm{c}} / \sigma_{\text {cluster }} \sim 0.5 \mathrm{Gyr}$, suggesting that most of these cluster galaxies may consume all of their gas within a single orbit around the cluster center, and form a passive cluster core by $z \sim 2$.

It should be noted that because the sensitivity of the $\mathrm{CO}(1-0)$ observation decreases toward larger radius from the cluster center (phase center), only gas-rich systems can be detected at large radii. However, as shown in Figure 1, our $\mathrm{CO}(1-0)$ detected sample comprise a mass-complete sample of cluster member galaxies; i.e., we are not missing massive star-forming yet gas-poor galaxies up to $2 \times$ FWHM. Hence, our result is not affected by this observational bias.

\section{Discussion and Conclusions}

We have obtained $\mathrm{CO}(1-0)$ for 14 massive SFGs in the $z=2.51$ cluster CLJ1001, the largest sample of galaxies within a single cluster with gas content constraints at $z>2$. These COdetected galaxies include nearly all of the massive SFGs within $2 R_{200 \text { c }}$, enabling a highly complete census of gas content in massive cluster galaxies. Here we first summarize our main results and then discuss their implications for the formation of massive galaxies in clusters. Our main results are summarized as follows.

1. Our cluster galaxies exhibit large differences in their star formation activity and gas masses: some are gas-poor, lowSFR, while others are gas-rich, starbursting systems. We show that this large variety of properties mainly correlates with the location of galaxies in the cluster (e.g., with their distance from the cluster core and their accretion state). This is particularly clear when considering the phase-space diagram, which shows that while galaxies remain relatively gas-rich when they first enter the cluster, their gas content is rapidly reduced as they approach the cluster center (enter the virial radius).

2. Despite their varieties in gas content and SFR, most cluster galaxies are found to exhibit elevated SFE with a typical gas depletion time of $\sim 400 \mathrm{Myr}$. This gas depletion time is comparable to the dynamical time of the cluster, suggesting that most galaxies may lose their gas (and become quiescent) within a single round-up around the center of the cluster, as further supported by the absence of gas-rich galaxies in the core of this young cluster.

The strong dependence on a clustercentric radius of gas content and SFE for these massive SFGs provide evidence that the dense environment plays an important role in shaping the formation/evolution of the most massive cluster galaxies. The significant suppression of molecular gas for all of the massive cluster galaxies close to the center (within virial radius) is a direct indication that environmental effects helping to stop gas accretion and/or reduce/remove gas content must have taken place. Various mechanisms have been proposed in the literature that reduce the gas content of cluster member galaxies such as starvation (namely, further gas accretion is stopped) or ram pressure and tidal stripping (the gas is removed from the galaxies). The rapid transition between gas-rich and gas-poor systems in the cluster takes place close to the cluster center with $k_{b} \sim 0.1$, supporting the idea that the main mechanisms involved may be ram pressure and tidal stripping, which happen close to the deep cluster potential (Treu et al. 2003). Moreover, the short gas depletion timescale ( $\sim 0.4 \mathrm{Gyr}$, comparable to the dynamical time of the cluster) is also consistent with simulations showing that ram pressure stripping could remove all of the gas of cluster members within a single radial orbit around the cluster center (Cen 2014). While current observations suffer from relatively poor resolution, future deep high-resolution observations of both stellar and gas distribution, as well as kinematics, would provide more insights into the main environmental mechanisms at work in this young cluster.

We thank the referee for very constructive comments that helped to improve the quality and clarity of this Letter. We thank Zhi-Yu Zhang for useful discussion on data reduction. This Letter makes use of the data from Karl G. Jansky Very Large Array, operated by the National Radio Astronomy Observatory. This Letter makes use of the following ALMA data: ADS/JAO.ALMA\#2016.01155.S and \#2011.0.00539. S. ALMA is a partnership of ESO (representing its member states), NSF (USA) and NINS (Japan), together with NRC (Canada), NSC and ASIAA (Taiwan), and KASI (Republic of Korea), in cooperation with the Republic of Chile. The Joint ALMA Observatory is operated by ESO, AUI/NRAO and NAOJ. This study was supported by the JSPS Grant-in-Aid for Scientific Research (S) JP17H06130 and the NAOJ ALMA Scientific Research grant No. 2017-06B. T.W. acknowledges support by the the European Commission through the FP7 SPACE project ASTRODEEP (ref. No. 312725).

Facilities: VLA, HST, Subaru.

\section{ORCID iDs}

Tao Wang (1) https://orcid.org/0000-0002-2504-2421 Emanuele Daddi (i) https://orcid.org/0000-0002-3331-9590 Daizhong Liu (iD https://orcid.org/0000-0001-9773-7479 Tadayuki Kodama (i) https://orcid.org/0000-0002-2993-1576 Ichi Tanaka (iD https://orcid.org/0000-0002-4937-4738 Corentin Schreiber (1) https://orcid.org/0000-0003-0942-5198 Anita Zanella (i) https://orcid.org/0000-0001-8600-7008 Francesco Valentino (i) https://orcid.org/0000-00016477-4011

Mark Sargent (ib https://orcid.org/0000-0003-1033-9684

Kotaro Kohno (iD https://orcid.org/0000-0002-4052-2394

Maurilio Pannella (1) https://orcid.org/0000-0003-3738-3976

Raphael Gobat (D) https://orcid.org/0000-0003-0121-6113

\section{References}

Aravena, M., Carilli, C. L., Salvato, M., et al. 2012, MNRAS, 426, 258 Bolatto, A. D., Wolfire, M., \& Leroy, A. K. 2013, ARA\&A, 51, 207 Boselli, A., \& Gavazzi, G. 2006, PASP, 118, 517

Bussmann, R. S., Riechers, D., Fialkov, A., et al. 2015, ApJ, 812, 43 
Casasola, V., Magrini, L., Combes, F., et al. 2013, A\&A, 558, A60

Casey, C. M. 2016, ApJ, 824, 36

Casey, C. M., Cooray, A., Capak, P., et al. 2015, ApJL, 808, L33

Cen, R. 2014, ApJ, 781, 38

Chabrier, G. 2003, PASP, 115, 763

Coogan, R. T., Daddi, E., Sargent, M. T., et al. 2018, MNRAS, 479, 703

Cortese, L., Davies, J. I., Pohlen, M., et al. 2010, A\&A, 518, L49

Dannerbauer, H., Lehnert, M. D., Emonts, B. H. C., et al. 2017, A\&A, 608, A48

Davis, T. A., Alatalo, K., Bureau, M., et al. 2013, MNRAS, 429, 534

Delhaize, J., Smolčić, V., Delvecchio, I., et al. 2017, A\&A, 602, A4

Dressler, A., Oemler, A., Jr., Couch, W. J., et al. 1997, ApJ, 490, 577

Genzel, R., Tacconi, L. J., Lutz, D., et al. 2015, ApJ, 800, 20

Hayashi, M., Kodama, T., Kohno, K., et al. 2017, ApJL, 841, L21

Hayashi, M., Kodama, T., Tanaka, I., et al. 2016, ApJL, 826, L28

Jáchym, P., Combes, F., Cortese, L., Sun, M., \& Kenney, J. D. P. 2014, ApJ, 792,11

Jáchym, P., Sun, M., Kenney, J. D. P., et al. 2017, ApJ, 839, 114

Kennicutt, R. C., Jr. 1998, ARA\&A, 36, 189

Koyama, Y., Kodama, T., Tadaki, K.-i., et al. 2013, MNRAS, 428, 1551

Laigle, C., McCracken, H. J., Ilbert, O., et al. 2016, ApJS, 224, 24

Lee, M. M., Tanaka, I., Kawabe, R., et al. 2017, ApJ, 842, 55

Magdis, G. E., Daddi, E., Béthermin, M., et al. 2012, ApJ, 760, 6

McCracken, H. J., Milvang-Jensen, B., Dunlop, J., et al. 2012, A\&A, 544, A156
McMullin, J. P., Waters, B., Schiebel, D., Young, W., \& Golap, K. 2007, in ASP Conf. Ser. 376, Astronomical Data Analysis Software and Systems XVI, ed. R. A. Shaw, F. Hill, \& D. J. Bell (San Francisco, CA: ASP), 127 Muzzin, A., Marchesini, D., Stefanon, M., et al. 2013, ApJS, 206, 8

Noble, A. G., McDonald, M., Muzzin, A., et al. 2017, ApJL, 842, L21

Noble, A. G., Webb, T. M. A., Muzzin, A., et al. 2013, ApJ, 768, 118

Rudnick, G., Hodge, J., Walter, F., et al. 2017, ApJ, 849, 27

Sargent, M. T., Daddi, E., Béthermin, M., et al. 2014, ApJ, 793, 19

Schreiber, C., Elbaz, D., Pannella, M., et al. 2018, A\&A, 609, A30

Schreiber, C., Pannella, M., Elbaz, D., et al. 2015, A\&A, 575, A74

Shimakawa, R., Kodama, T., Hayashi, M., et al. 2017, arXiv:1708.06369

Silverman, J. D., Daddi, E., Rodighiero, G., et al. 2015, ApJL, 812, L23

Smolčić, V., Novak, M., Bondi, M., et al. 2017, A\&A, 602, A1

Solomon, P. M., \& Vanden Bout, P. A. 2005, ARA\&A, 43, 677

Stach, S. M., Swinbank, A. M., Smail, I., Hilton, M., \& Simpson, J. M. 2017, ApJ, 849, 154

Tacconi, L. J., Genzel, R., Saintonge, A., et al. 2018, ApJ, 853, 179

Tadaki, K.-i., Kodama, T., Tamura, Y., et al. 2014, ApJL, 788, L23

Thomas, D., Maraston, C., Bender, R., \& Mendes de Oliveira, C. 2005, ApJ, 621,673

Treu, T., Ellis, R. S., Kneib, J.-P., et al. 2003, ApJ, 591, 53

Wagg, J., Pope, A., Alberts, S., et al. 2012, ApJ, 752, 91

Wang, T., Elbaz, D., Daddi, E., et al. 2016, ApJ, 828, 56

Wetzel, A. R., Tinker, J. L., Conroy, C., \& van den Bosch, F. C. 2013, MNRAS, 432, 336 